\title{
Clinical Study of Cholesteatoma in Otitis Media
}

\author{
Dr. Preeti Sharma ${ }^{1}$, Dr. P.N.P Pal ${ }^{2}$ \\ ${ }^{1,2}$ Department of ENT, Patna Medical College and Hospital, Patna
}

\begin{abstract}
:
Introduction: The introduction of the otomicroscope in ear surgery has undoubtedly resulted in a better and more scientific approach to ear disease and has also added a fresh dimension to the treatment of chronic suppurative otitis media i.e. reconstruction of hearing mechanism. Although western reports indicate that the rate of cholesteatomatous complications is as low as 1 or 2 percent, it remains a significant cause of morbidity and even mortality in our country.

Material and methods: This study included fifty cases of chronic supprative otitis media with cholesteatoma. In all cases detailed history were obtained and Otoscopic, otomicroscopic and tunning fork tests were performed. $X$-ray mastoids (Schuller's view) and CT scan was done for patients with complications.

Result: Chronic otitis media (COM) commonly occurs in the age group from 11 to 30 years (76\%). Otorrhea was the main reason for consultation (100\%). Destruction of incus was the commonest Intraoperative finding (76\%). Malleus was absent in (30\%) while stapes superstructure was eroded in (40\%). Intracranial complications were seen in $28 \%$ whereas $18 \%$ cases presented with extra cranial complications.

Conclusion: Chronic otitis media is prevalent in lower socio-economic strata. Patients often present late with advanced disease process associated with complications. Meticulous clinical examination should be done and surgery should be accordingly planned.
\end{abstract}

Keywords: chronic suppurative otitis media, cholesteatoma, otorrhea, complications

\section{Introduction}

Chronic suppurative otitis media remain a major problem in developing countries like India due to poverty, crowding, illiteracy and poor hygiene conditions. Although the potential threat of ear disease has been known from centuries, rational treatment has evolved only over the last century. The advent of antimicrobial has greatly altered the course of the disease and drastically reduced complications. The introduction of the otomicroscope in ear surgery has undoubtedly resulted in a better and more scientific approach to ear disease and has also added a fresh dimension to the treatment of chronic suppurative otitis media i.e. reconstruction of hearing mechanism.

Although western reports indicate that the rate of cholesteatomatous complications is as low as 1 or 2 percent, it remains a significant cause of morbidity and even mortality in our country. In our present state our main objective should be reduce the incidence of complications. Reconstructive otologic surgery only comes secondary to the above goal. The objective of our study is to probe the clinical profile of cholesteatoma under local conditions.

Chronic suppurative otitis media is defined as a persistent or intermittent infected discharge through a non-intact tympanic membrane. Chronic perforation of the tympanic membrane can occur without suppuration and is often referred to as "inactive" CSOM. CSOM is particularly prevalent in developing countries and is more common in lower socioeconomic groups in the developed world.

CSOM is the result of non resolution of acute suppurative OM. Acute infection that does not resolve may produce a sub acute condition with continuing otorrhoea; possibly from a mastoid reservoir. CSOM can be grouped into two main clinical types: safe type and dangerous type.

The safe type is so called because it carries limited risk to the patient of any serious complications. The infective disease is limited to mucosa and to the antero-inferior part of the middle ear cleft - for which reason it is sometimes described as tubotympanic. The dangerous type of disease is associated with erosion of bone, and the chance of exposing to infection, the important structures within the temporal bone, or within the skull. This type of disease involves the postero-superior part of the middle ear cleft "attico-antral region", therefore terming it is as atticoantral disease. In these circumstances the perforation through which the discharge emanates is either in the attic, or in the posterior region of the pars tensa, with no drum/ annulus to separate it from the posterior bony canal wall - so called marginal perforation.

German physiologist Johannes Mueller coined the term cholesteatoma in 1936. Term Cholesteatoma is a great misnomer in otology because although it behaves like a tumor it is neither a tumor "oma", nor does it contain cholesterin: "chole" or fat "stea". Tos ${ }^{1}$ later classified Cholesteatoma on the basis of the site of origin of Cholesteatoma, which is an important factor for surgical procedure and prognosis. Attic Cholesteatoma - 
Defined as retraction of pars flaccida or Shrapnel's membrane extending into attic or aditus and eventually into the antrum, mastoid or tympanic cavity. Sinus Cholesteatoma - Posterosuperior retraction or perforation of the pass tensa extending to the tympanic sinus, posterior tympanum and beyond. Tensa Cholesteatoma - Retraction and adhesion of the entire pars tensa involving the tympanic orifice of the Eustachian tube ${ }^{1,2}$

\section{Theories of Origin}

Squamous Metaplasia Theory -Wendt (1873): Wendt suggested that the non keratinizing epithelium of the middle ear and the mastoid could undergo a metaplastic transformation into keratinizing epithelium. The metaplastic epithelium grows because of the accumulation of keratin, and recurrent infection and inflammation leads to lysis and perforation of the tympanic membrane resulting in the typical appearance of attic cholesteatoma.

Immigration Theory - Haberman ${ }^{3}$ : Haberman in 1888 and Bezold in 1890 proposed the epithelial immigration or invasion theory suggesting that squamous epithelium migrates from the margin of a tympanic membrane perforation into the middle ear spaces ${ }^{4,5}$.

In 1901, Politzer observed that the epidermis of the external auditory canal can grow over a perforation of the tympanic membrane into the middle ear cavity. He assumed that after perforation of the tympanic membrane, a second infection would be the cause of epithelial migration.

Basal Hyperplasia Theory: Lange observed in 1925 that epithelial cells from the keratinizing epithelium of the pars flaccida could invade into the subepithelial space and form an attic cholesteatoma.

Retraction Pocket or Invagination Theory: This theory was presented by Wittmack in 1933 and is generally accepted as the most common pathogenetic mechanism in acquired cholesteatoma. According to this theory, the pars flaccida or occasionally the pars tensa of the tympanic membrane retracts into the middle ear may be due to negative pressure, inflammation or both. The retention of accumulating keratin within the deep retraction pocket establishes cholesteatoma. The loss of drainage of the retraction pocket is thought to induce the expansion into the middle ear and mastoid spaces.

\section{Pathology Of Cholesteatoma ${ }^{6}$}

Macroscopically, cholesteatoma appears as a roundish or oval shaped friable mass which is whitish' in color, pultaceous in consistency of a variable size ranging from a size of a walnut to even measuring over $5 \mathrm{~cm}$ in diameter.

Microscopically the lesion is made up of three components, i.e. the cystic content, the matrix and the perimatrix. The cystic content is composed of fully differentiated anucleate keratin squamous along with some purulent and necrotic matter in the cavity. The matrix and cholesteatoma consists of keratinizing squamous epithelium lining a cyst like structure. The perimatrix or lamina propria is the peripheral part of the cholesteatoma and consists of granulation tissue or inflamed subepithelial connective tissue displaying inflammatory cells composed and lymphocytes, histocytes, plasma cells and neutrophil leucocytes.

\section{Materials And Methods}

This study included fifty cases of chronic supprative otitis media with cholesteatoma. Cases of congenital cholesteatoma and residual cholesteatoma were excluded from this study.

In all cases detailed history were obtained. Otoscopic and otomicroscopic and tunning fork tests were performed. Routine investigations were done for all cases. Ear discharge was sent for culture and sensitivity. Xray mastoids (Schuller's view) were taken for all cases and CT scan was done for patients with complications. Pure tone audiometry was done for all cases.

All patients underwent mastoid exploration by the post aural route and were treated postoperatively with appropriate antibiotics. Most patients were discharged after a week following suture removal. Patients were then instructed to come for regular follow up for toileting of the mastoid cavity.

\section{Observation}

This study was based on 50 patients who underwent mastoid exploration for cholesteatomatous ear disease. In our study age group ranged from 8 to 50 yrs. Youngest patient was eight yrs and oldest was $50 \mathrm{yrs}$. old. Maximum incidence of cholesteatoma was seen in the second decade. In the present study there was a male predominance constituting almost $68 \%$ while females made up only $32 \%$ of the study.

Of the fifty cases studied, 15 (30\%) of them had bilateral cholesteatoma while the remaining $35(70 \%)$ had unilateral disease. Among these patients who had unilateral disease, 17 (34\%) of them had it on the right side while 18 (36\%) of them had it on the left side. Of the 35 cases which had unilateral ear disease, 4 of them had coexistent tubotympanic disease on other side. The minimum period of ear discharge encountered was 5 months and maximum was over 30 years duration. The majority of cases, almost $80 \%$ had chronic otorrhoea from 1 to 10 years duration. 
All the cases in this study presented with otorrhoea. 46 patients (92\%) had history of hearing loss. Otalgia was seen in 14 patients $(28 \%)$, fever in $7(14 \%)$, giddiness in $6(12 \%)$ and tinnitus was seen in $4(8 \%)$ cases. Vomiting was seen in $4(8 \%)$ patients and headache in $3(6 \%)$.

On microscopic examination, perforation was seen in the posterosuperior quadrant of the tympanic membrane in $27(54 \%)$ cases, while attic perforation was seen in $21(42 \%)$ cases. The remaining 2 cases $(4 \%)$ had a central perforation.

\begin{tabular}{|c|c|}
\hline Age Distribution & Number of patients (\%) \\
$0-10$ years & $4(8 \%)$ \\
$11-20$ years & $21(42 \%)$ \\
$21-30$ years & $17(34 \%)$ \\
$31-40$ years & $6(12 \%)$ \\
41 and above & $2(4 \%)$ \\
\hline Sex Distribution Male & $34(68 \%)$ \\
Female & $16(32 \%)$ \\
\hline Side Of Involvement & $15(30 \%)$ \\
Bilateral disease & $17(34 \%)$ \\
Right sided & $18(36 \%)$ \\
Left sided & $1(2 \%)$ \\
Duration of discharge & $4(8 \%)$ \\
0-6 months & $9(18 \%)$ \\
6 months to 1 year & $33(66 \%)$ \\
1 year - 3 years & $3(6 \%)$ \\
3 years - 10 years & \\
More than 10 years &
\end{tabular}

Table 1: Demographic profile

In all the cases X-ray of the mastoids, schuller's view was taken and was analyzed. They were classified as whether they were pneumatized or scelerosed. Some cases showed a well define cavity with in which the cholesteatoma sac was present. All the X-rays of involved ears showed sclerotic mastoid, 43 (86\%) of these patients showed cavity lesions in the X-rays. No patients had pneumatized $\mathrm{x}$-ray finding.

Microbiological profile showed Staphylococcus aureus as the commonest organism isolated in this series and was seen in 13 cases i.e. 26\%. This was followed by pseudomonas 11 (22\%), proteus 9 (18\%), klebsiella $3(6 \%)$, E.coli $1(2 \%)$ and Haemophilus influenza $1(2 \%)$ cases. Mixed growth was seen in 6 cases i.e. $12 \%$. No organism was isolated in 6 cases $(12 \%)$.

All the cases were subjected for PTA and hearing threshold for air conduction and bone conduction were noted. In this series, there were 27 cases with pure conductive hearing loss i.e. 54\%. Mixed loss was seen in 18 cases i.e. $36 \%$ and sensorineural loss in 5 cases i.e. $10 \%$.

\begin{tabular}{|c|c|c|}
\hline Type & No. of patients & \% age of patients \\
\hline Conductive & 27 & 54 \\
\hline Mixed & 18 & 36 \\
\hline Sensorineural & 5 & 10 \\
\hline
\end{tabular}

Table 2: Audiological profile hearing loss

The average air conduction threshold at $500,1 \mathrm{k}$ and $2 \mathrm{k} \mathrm{Hz}$ was noted in all cases and majority of them i.e. $64 \%$ (32 patients) had a hearing threshold between 31 to $50 \mathrm{db}$. About $10 \%$ i.e. 5 cases had their air conduction threshold of loss than $30 \mathrm{db}$ and $26 \%$ i.e 13 cases had a threshold of more than $60 \mathrm{db}$.

\begin{tabular}{|c|c|c|}
\hline A. C. Threshold & No. of patients & \% age of patients \\
\hline$<30 \mathrm{db}$ & 5 & 10 \\
\hline 31 to $59 \mathrm{db}$ & 32 & 64 \\
\hline$>60 \mathrm{db}$ & 13 & 26 \\
\hline
\end{tabular}

Table 3: Air conduction threshold

\section{Operative Findings}

All cases underwent inside out canal wall down and findings were noted. Destruction of the incus either part or as a whole was the commonest intraoperative finding noted in our study. This was seen in as many as 38 cases $(76 \%)$. Malleus was absent in 15 cases $(30 \%)$ while stapes superstructure was eroded in 20 cases $(40 \%)$. Erosion of the facial nerve canal was noted in as many as 9 cases $(18 \%)$. Erosion of the dural and the 
sinus plate was seen in $3(6 \%)$ and $4(8 \%)$ cases respectively. Erosion of the lateral semicircular canal bulge was seen in 3 cases i.e. $6 \%$, but the membranous labyrinth was open in only one case. This patient had a dead ear.

\begin{tabular}{|c|c|c|}
\hline Finding No. of & No. of patients & \% age of patients \\
\hline Incus absent & 38 & 76 \\
\hline Malleus absent & 15 & 30 \\
\hline Stapes superstructure absent & 20 & 40 \\
\hline Facial canal erosion & 9 & 18 \\
\hline Dural plate erosion & 3 & 6 \\
\hline Sinus plate erosion & 4 & 8 \\
\hline Lateral semicircular canal erosion & 3 & 6 \\
\hline
\end{tabular}

Table 4: Operative Findings

Types of complication: In this study of fifty cases of cholesteatoma, we noticed complications in 14 cases i.e. $28 \%$. Of these 9 of them i.e. $18 \%$ were extra cranial complications while the remaining 5 cases i.e. $8 \%$ were intra cranial.

\begin{tabular}{|c|c|c|}
\hline & No. of patients & \% age of patients \\
\hline Extra cranial & & 16 \\
Mastoid abscess & 8 & 2 \\
Facial nerve paralysis & 1 & 4 \\
\hline Intra Cranial & 2 & 2 \\
Meningitis & 1 & 4 \\
Brain abscess & 2 & \\
Extra dural abscess & & \\
\hline
\end{tabular}

Table 5: complications observed

All patients were eventually subjected to surgery. The mastoid was explored by the post aural route. A canal wall down was adopted in all cases. Attic cholesteatomas with small sclerotic mastoid were dealt with inside out mastoidectomy (30\%). Most cases underwent a modified radical mastoidectomy i.e. $70 \%$.

\begin{tabular}{|c|c|c|}
\hline Type of Surgery & No. of patients & \% age of patients \\
\hline Inside out mastoidectomy & 15 & 30 \\
\hline Modified radical mastoidectomy & 35 & 70 \\
\hline
\end{tabular}

Table 6: Type of surgery

\section{Discussion}

The commonest presenting symptom in our study was otorrhoea found in all cases followed by hearing loss $(92 \%)$. Edelstein et $\mathrm{al}^{7}$ found hearing loss as the commonest feature followed by otorrhoea (75\%). Incidence of other clinical features such as otalgia (28\%), vertigo (12\%) and tinnitus (8\%) corresponds to findings of Edelstein et al. incidence of marginal perforation was found to be $32 \%$ while attic and central perforation was found in $20 \%$ and $9 \%$ cases respectively.

Intraoperatively, necrosed incus was found in $76 \%$ cases which was higher than Edelstein et al (46\%) and in accordance to Sade et al. ${ }^{8}$ In our study malleus and stapes necrosis was foud in $30 \%$ and $40 \%$ respectively which was in accordance with other studies. Dural plate erosion (6\%) was found as intra-operative finding $\mathrm{n}$ our study but not in study of Edelstein et al. higher incidence of bone erosion seen in our study might be due to delay on patient's part in seeking medical advice and intervention.

Intra-cranial as well as extra-cranial complications (10\% and $18 \%$ respectively) were higher in comparison to Gupta et $\mathrm{al}^{9}$ ( $0.82 \%$ and $2.38 \%$ respectively). Higher complication rate in our study might be due to more patients of rural background who neglect their disease for long time.

\section{Conclusion}

The disease was predominant in people belonging to lower socio-economic class with most common symptoms being chronic ear discharge. Although bilateral cholesteatoma is reported in $30 \%$ of cases and unilateral cases shows almost equal distribution with left $(36 \%)$ and right $(34 \%)$ of the cases in the present study. Posterosuperior and attic cholesteatoma was found in $54 \%$ and $42 \%$ respectively.

The average hearing loss ranged from 20-40 dB. Greater degrees of conductive loss were seen in patients with secondary acquired cholesteatoma. Patients with attic retraction cholesteatoma had an average loss of 20-25 dB in most cases. Patients with intracranial complications were accordingly treated with appropriate antibiotics and brain abscess was drained wherever required. 
All patients were subjected to surgery. Canal well down was adopted in all cases. Attic cholesteatomas with small sclerotic mastoid were dealt by inside and mastoidectomy. Patients with extensive cholesteatoma or granulations in the middle ear were treated by modified radical mastoidectomy. In our study 15 patients i.e. $30 \%$ underwent inside out mastoidectomy and 35 patients i.e. $70 \%$ underwent modified radical mastoidectomy.

\section{References:}

[1]. Lau and M. Tos, "Tensa retraction cholesteatoma: treatment and long-term results," Journal of Laryngology and Otology, vol. 103, no. 2, pp. 149-157, 1989.

[2]. H. A. Saleh and R. P. Mills, "Classification and staging of cholesteatoma" Clinical Otolaryngology and Allied Sciences, vol. 24, no. 4, pp. 355-359, 1999

[3]. Wullstein H. theory and practice of tympanoplasty. Laryngoscope 1956; 66; 1076-1093

[4]. E. Olszewska, M. Wagner, M. Bernal-Sprekelsen et al., "Etiopathogenesis of cholesteatoma," European Archives of Oto-Rhino-Laryngology, vol. 261, no. 1, pp. 6-24, 2004.

[5]. Scott-Brown's. Chronic otitis media. In : Otorhinolaryngology, Head and Neck Surgery. Gleeson M, Browning GG, Burton MJ, Clarke R, Hibbert J, Jones NS, et a, eds. Chapter 237, $7^{\text {th }}$ edn. Vol. 3 2008. P. 3395-3397

[6]. D. Soldati and A. Mudry, "Knowledge about cholesteatoma, from the first description to the modern histopathology," Otology \& Neurotology, vol. 22, no. 6, pp. 723-730, 2001.

[7]. Edelstein DR, Parisier SC. Surgical techniques and recidivism in cholesteatoma. Otolaryngol Clin North Am. 1989;22:1029-1040

[8]. Sade J, Babiacki A, Pinkos G. The Metaplastic and congenital origin of cholesteatoma. Acta Otolaryngol 1983; 96 : Pg. 119-129.

[9]. Gupta A, Agarwal SR. A study of prevalence of cholesteatoma in complications of otitis media. Indian journal of otolaryngology \& head and neck surgery 1998; 50:140-146 